\title{
Aprendizaje de la seguridad asistencial en medicina intensiva
}

\author{
Patient safety's learning in intensive medicine
}

\author{
Carolina Ruiz Balart ${ }^{1}$, Denisse Zúñiga Parada²
}

\begin{abstract}
Resumen
Introducción: la seguridad asistencial (SA) es una competencia especialmente relevante en las Unidades de Cuidados Intensivos (UCI), ya que la gravedad de los pacientes y los tratamientos que reciben, generan situaciones de riesgo para eventos adversos (EA). Se presenta una revisión narrativa de los conceptos más importantes de SA, con énfasis en su enseñanza-aprendizaje en medicina intensiva (MI). Además, se describen los resultados de una experiencia piloto sobre formación en SA, para residentes de MI de la Pontificia Universidad Católica de Chile (UC). Métodos: para la revisión narrativa se utilizó una estrategia de búsqueda amplia en Pubmed, considerando artículos clínicos y de educación. También se revisó la literatura nacional, incluyendo libros de resúmenes de congresos. La experiencia piloto consistió en un taller de cinco sesiones, basado en aprendizaje en pequeño grupo. Resultados: iniciativas internacionales han tratado de estandarizar el aprendizaje de la SA. Para una atención de salud segura deben considerarse competencias como comunicación, liderazgo y trabajo en equipo; pues, 20-30\% de los EAs están relacionados con estas. Al término del taller de formación en SA, se encontró que la adquisición de conocimientos fue adecuada; sin embargo, no hubo mejoría en la percepción de la importancia de esta competencia. Conclusión: la SA es fundamental para cualquier actividad clínica, siendo indispensable la capacitación de los intensivistas en esta competencia. La próxima versión del dispositivo de formación en SA para residentes de la subespecialidad de MI de la UC se optimizará, agregando un módulo de aprendizaje en el lugar de trabajo en la UCl.
\end{abstract}

Palabras clave: seguridad asistencial; cultura de la seguridad; enfermedad crítica; unidad de cuidados intensivos; evaluación; residentes

\begin{abstract}
Introduction: Patient Safety (PS) is a relevant competence in the Intensive Care Unit (ICU) since the severity of the patients and the treatments they receive generate risk situations for Adverse Events (AE). A narrative review of the most important concepts of PS is presented, with emphasis on its teaching-learning process in the area of intensive medicine (IM). Also, the results of a PS training pilot program for IM residents of Pontificia Universidad Católica de Chile (UC), are described. Methods: For the literature review, a broad search strategy in PubMed was used, including publications in English and Spanish; clinical and educational articles were considered. Local, national literature was also reviewed, including conference abstract-books. The pilot program consisted of a five-session workshop, based on a small group learning strategy. Results: International initiatives have tried to standardize PS learning. Other competencies, such as communication, leadership, and teamwork, must also be considered for safe healthcare, as $20-30 \%$ of AE are related to these. At the end of the IM-resident PS workshop, it was found that knowledge acquisition was adequate; however, there was no improvement in the perception of the importance of PS. Conclusion: PS is essential for any clinical activity, and training in it is mandatory for intensivists. The next version of the PS training program for IM residents of UC will be optimized by adding a workplace-based learning module in the ICU.
\end{abstract}

Keywords: patient safety; safety culture; critical illness; intensive care unit; evaluation; residents.

Fecha de envío: 2020-06-07 - Fecha de aceptación: 2020-11-30

\section{Introducción}

La seguridad asistencial (SA) consiste en la libertad de daño o de potencial daño asociado con la atención sanitaria, siendo, actualmente, uno de los temas más importantes en la agenda de salud a nivel mundial (World Health Organization, 2017). Este daño asociado con la atención en salud puede generar importantes

(1) Departamento de Medicina Intensiva, Escuela de Medicina, Facultad de Medicina, Pontificia Universidad Católica de Chile.

(2) Centro de Educación Médica, Escuela de Medicina, Facultad de Medicina, Pontificia Universidad Católica de Chile

Autor de correspondencia: ccruiz@uc.cl 
complicaciones en los pacientes, que no se explican por la evolución natural de la enfermedad o por los efectos conocidos de los tratamientos recibidos, pudiendo incluso llegar a la muerte. Además, los profesionales de la salud (PS) que atienden a un paciente cuando ocurre un evento adverso (EA) y las instituciones donde este se produce pueden sufrir consecuencias negativas debido al EA. Ante esto, la Organización Mundial de la Salud (OMS) ha impulsado políticas internacionales para una atención de salud más segura. Dentro de estas, la capacitación en SA de todos los PS se considera fundamental e imprescindible (World Health Organization, 2011).

Entre 20 a 30\% de los EAs están relacionados con problemas de comunicación, liderazgo y/o trabajo en equipo; lográndose una disminución de estos EAs al entrenar estas competencias (Leonard et al., 2004; Manser, 2009). Sin embargo, la SA y las otras competencias necesarias para una atención de calidad han sido insuficientemente abordadas en la formación de los médicos, tanto a nivel de pregrado como de postgrado (Bagian, 2015).

La medicina intensiva (MI) es la subespecialidad encargada de proveer la atención y cuidado a los pacientes críticos. La SA es especialmente relevante en $\mathrm{MI}$, ya que la gravedad de los pacientes, junto con el tipo de trabajo y atención, generan situaciones de riesgo que pueden facilitar la ocurrencia de EAs. Debido a esto, varias iniciativas internacionales han tratado de estandarizar el aprendizaje de la SA en MI (Royal College of Physicians and Surgeons of Canada, 2014; Sociedad Europea de Medicina Intensiva, 2006).

A continuación, se presenta una revisión narrativa de los conceptos más importantes de SA, haciendo énfasis en su enseñanza-aprendizaje en MI. Además, se presentan los resultados de una experiencia piloto sobre formación en SA para residentes de MI del adulto de la Pontificia Universidad Católica de Chile (UC), utilizando el modelo de evaluación de programas educacionales de Kirkpatrick. Tanto la revisión de la literatura como la experiencia piloto forman parte de una tesis de Magíster en Educación Médica de la UC (Ruiz, 2019).

Para la revisión narrativa se revisó la literatura, utilizando una estrategia de búsqueda amplia en Pubmed, incluyendo publicaciones en inglés y español. Se consideraron artículos clínicos y de educación médica. También se revisó la literatura nacional, incluyendo libros de resúmenes de congresos.

\section{Seguridad asistencial: conceptos fundamentales y relevancia}

Durante los últimos años, la preocupación por la seguridad del paciente o seguridad asistencial ha sido un tema prioritario en salud en todo el mundo. En consecuencia, se han desarrollado políticas internacionales lideradas por la OMS que, junto con el esfuerzo mancomunado de instituciones sanitarias, organismos gubernamentales, PS y pacientes, han tenido por objetivo evitar/ limitar el potencial daño asociado con la atención sanitaria. Este daño es consecuencia de los errores del personal y de los sistemas de salud, así como de los riesgos sanitarios. Asimismo, esta preocupación global por la SA ha generado que en muchos países se dicten leyes al respecto. En Chile en el 2012 se promulgó la Ley 20584, que definió los derechos y deberes de los pacientes, estableciendo el derecho a una atención de salud segura (Ley de Derechos y Deberes de los Pacientes, 2012).

A nivel mundial, aproximadamente $10 \%$ de los pacientes ingresados a un hospital sufren un EA, destacando los relacionados con la administración de medicamentos, caídas, procedimientos, equipamiento médico, infecciones asociadas a la atención en salud, etc. (World Health Organization, 2017). Por otra parte, $10 \%$ de los EAs se asociarían a mortalidad, reportándose que 98.000 pacientes fallecen por año en EEUU debido a estos (Kohn et al., 2000). En Chile, las cifras de EAs también son cercanas al 10\% (Lancis \& Asenjo, 2014). Al respecto, una fiscalización realizada en nuestro país en el 2017 sobre EAs y eventos centinela, encontró que solo en $62 \%$ de los casos se cumplían las medidas de prevención necesarias (Superintendencia de Salud, 2017).

Dentro de la enseñanza-aprendizaje de la SA hay varios conceptos necesarios de revisar (tabla 1), destacando la cultura de la seguridad, que junto con la Teoría del Error de Reason son los fundamentos de la SA (Flott et al., 2017). La teoría del error señala que el ser humano es falible y que, pese a los esfuerzos por evitarlos, los errores seguirán produciéndose, siendo imposible eliminarlos. Hasta el PS más capacitado y competente puede cometer un error, ya sea por sobrecarga laboral, cansancio, problemas personales, complejidad de los pacientes, realización de múltiples intervenciones, etc. La cultura de la seguridad considera una visión no punitiva de los errores, en la que estos son aprovechados para aprender en vez de ser utilizados para culpabilizar, generándose un ambiente abierto y de confianza, conocido como clima de seguridad. Además, considera un enfoque sistémico del error y el riesgo. Según este enfoque, cuando un EA ocurre no es culpa de una sola persona, por el contrario, implica una falla de la organización sanitaria que debe revisarse. Al ser la conducta humana falible, los errores inevitables y los riesgos inherentes a la atención sanitaria, las organizaciones (centros asistenciales) y Ios PS deben utilizar protocolos que permitan asegurar la aplicación de las mejores prácticas y estándares de atención, junto con sistemas de búsqueda y control del error. Esta actitud proactiva frente a los incidentes de seguridad y EAs es clave en la mejora continua de la atención, que es otro elemento importante de la cultura de la seguridad (Kohn et al., 2000). 
La gestión del riesgo, que es uno de los componentes de la cultura de la seguridad, incluye los sistemas de reporte de incidentes de seguridad y los métodos de análisis causa-raíz. Los primeros son procedimientos estructurados de recolección, reporte y análisis de incidentes de seguridad. Los segundos consisten en distintos métodos para la resolución e idealmente prevención de problemas, que intentan evitar la recurrencia de estos al identificar sus causas, constituyendo procesos reiterativos y herramientas de mejora continua (World Health Organization, 2009). La gestión del riesgo es fundamental en la atención sanitaria, pues en los últimos años han aumentado los riesgos de esta. Esto se explica por la complejización del sistema de salud, el envejecimiento de la población y el aumento de las enfermedades crónicas, que constituyen factores de riesgo para incidentes de seguridad. Es importante tener en cuenta que mientras más complejo e invasivo sea un tratamiento, especialmente en pacientes mayores y con importantes comorbilidades, hay más riesgos de EAs. Por otra parte, esta complejización de la medicina muchas veces genera sobrecarga laboral para los PS, trabajo en condiciones de estrés y deshumanización de la atención sanitaria, los que también implican riesgos de errores.

En el entendido de que los EAs corresponden a fenómenos sistémicos, que implican fallas en varias etapas del proceso sanitario, la cultura de la seguridad reconoce tres tipos de víctimas de estos: los pacientes, los PS y los centros asistenciales (tabla 1). Estas tres víctimas tienen complicaciones y problemas tras la ocurrencia de un EA. La primera víctima puede tener múltiples complicaciones, incluyendo la prolongación de la hospitalización, traslados a unidades de alta complejidad, mayores gastos, nuevos tratamientos e incluso la muerte. La segunda víctima puede tener problemas laborales, legales, con sus pares, con los familiares de los pacientes e incluso pérdida de autoestima y depresión; existen casos emblemáticos y muy tristes de PS que, tras participar en un EA, han llegado incluso a quitarse la vida. La tercera víctima puede tener problemas legales, tener que pagar cuantiosas indemnizaciones, ver comprometido su prestigio, etc. (World Health Organization, 2009).

Tabla 1: Principales conceptos sobre seguridad asistencial.

\begin{tabular}{|c|c|}
\hline Concepto & Definición \\
\hline Error & $\begin{array}{l}\text { Es el hecho de no llevar a cabo una acción según corresponde, sin implicar necesariamente intencionalidad. Los errores } \\
\text { pueden manifestarse al hacer algo erróneo (error de comisión) o al no hacer lo correcto (error por omisión). }\end{array}$ \\
\hline $\begin{array}{l}\text { Incumplimiento o } \\
\text { infracciones }\end{array}$ & Desvío deliberado de las normas, reglas o procedimientos operativos. \\
\hline Riesgo sanitario & $\begin{array}{l}\text { Probabilidad de que un paciente presente un evento adverso. Está relacionado con factores del paciente (gravedad, co- } \\
\text { morbilidades, edad, etc.) y de la atención sanitaria (personal poco capacitado, procedimientos complejos e invasivos, etc.). } \\
\text { Los errores potencian el riesgo sanitario. }\end{array}$ \\
\hline Incidente de seguridad & $\begin{array}{l}\text { Circunstancia no intencional que le ocurre a un paciente en un centro asistencial y que le pueden ocasionar daño, el que } \\
\text { no puede explicarse por la evolución natural de la enfermedad o por los riesgos conocidos de un tratamiento. }\end{array}$ \\
\hline Evento adverso & Incidentes de seguridad que producen daño a un paciente. \\
\hline Evento centinela & Es un EA que produjo o pudo producir la muerte o una lesión grave, ya sea física o psíquica, a un paciente. \\
\hline Cultura de la seguridad & $\begin{array}{l}\text { Es un patrón de comportamiento individual y organizacional que busca continuamente reducir al mínimo el daño que } \\
\text { podría sufrir un paciente como consecuencia de la atención sanitaria. }\end{array}$ \\
\hline Gestión del riesgo & $\begin{array}{l}\text { Componente de la cultura de la seguridad que consiste en un conjunto de actividades destinadas a identificar, evaluar y } \\
\text { reducir o eliminar el riesgo de que se produzca un EA. Dentro de estas actividades destacan los sistemas de chequeo y } \\
\text { control del error. }\end{array}$ \\
\hline Primera víctima & $\begin{array}{l}\text { Corresponde a un paciente que sufrió un EA. Algunos autores señalan que los familiares también podrían ser considerados } \\
\text { dentro de las primeras víctimas. }\end{array}$ \\
\hline Segunda víctima & PS que estuvo involucrado en la atención de un paciente durante un EA. \\
\hline Tercera víctima & Es la institución de salud donde ocurrió el EA. \\
\hline
\end{tabular}

Abreviaciones: SA (seguridada asistencial), EA (evento adverso) 


\section{Formación de los médicos en seguridad asistencial}

Se ha reconocido la importancia de educar a los estudiantes de las carreras de la salud sobre los principios y conceptos de la seguridad del paciente, asimismo, en otras competencias transversales relacionadas con esta. La SA corresponde a una competencia y, por lo tanto, se puede enseñar, entrenar y evaluar. Una competencia se define como un desempeño observable, que requiere de la integración de conocimientos, destrezas y actitudes (Risco de Domínguez, 2014). La OMS ha manifestado en varias oportunidades la necesidad de que, en los currículos de pre y postgrado de las distintas carreras de la salud, incluida medicina, se considere la formación en esta competencia (World Health Organization, 2011). Esta necesidad fue ratificada recientemente por la OMS en el documento Medidas Mundiales en Materia de Seguridad del Paciente, que señala: "la seguridad del paciente se basa en una educación y formación básica y continua de calidad de los profesionales de la salud, con el fin de garantizar que tengan las aptitudes y competencias profesionales adecuadas en sus respectivos papeles y funciones" (Organización Mundial de la salud, 2019).

Varios autores han manifestado que la SA es insuficientemente abordada en la formación de los médicos, no obstante, su importancia, en pregrado y en postgrado (Bagian, 2015). Esto se ha atribuído a distintas razones, destacando la falta de reconocimiento por parte de los médicos de que la SA es esencial en su rol profesional, al igual que, la renuencia a considerar conceptos que fueron desarrollados fuera de la medicina, como las nociones de mejora continua de la calidad y la seguridad. Además, la profesión médica se ha centrado tradicionalmente en el tratamiento de las enfermedades y no en la prevención o en los cuidados, a diferencia de otros PS más familiarizados con la cultura de la seguridad. En este sentido, se ha planteado que será difícil que la SA mejore sin reformas profundas a los currículos de pre y postgrado de la carrera de medicina a nivel global (Vivekananda-Schimdt et al., 2016).

La cultura de la seguridad considera en la prevención y control precoz de los errores muchas de las estrategias que han demostrado ser efectivas en la aviación comercial, área que ha logrado posicionarse como un líder mundial en seguridad. Dentro de estas estrategias destaca el reforzamiento de la comunicación, el liderazgo y el trabajo en equipo. Reforzar estas competencias en la formación de los médicos se ha asociado con una mejoría de la valoración de la SA, asimismo, con una reducción de los errores y EAs (Leonard et al., 2004; Manser, 2009). La formación en SA y en las otras competencias transversales relacionadas con esta, son especialmente importantes en determinadas especialidades y subespecialidades, como las especialidades quirúrgicas, la anestesia y la MI. Las condiciones de trabajo y las caracteríticas de los pacientes atendidos por estas especialidades, hacen que estos tengan un riego importante de presentar EAs.
Por último, la necesidad de formación en SA de los médicos y otros PS también ha estado motivada por la relación de esta competencia con la bioética y el profesionalismo. Dentro de los cuatro principios bioéticos del Modelo de Beauchamp y Childress (Couceiro \& Muñoz, 2008), el principio de la no maleficencia siempre debe respetarse. Los PS y las instituciones sanitarias no estarían cumpliendo con este imperativo ético si no se preocupan de minimizar el error y así evitar el daño asociado a los EAs. El profesionalismo es una competencia fundamental en la formación de los médicos y ha sido definido como del Modelo Principialista cos e cita distintos autores, de competecias no tonsideraciSA. de aprendizaje sobre SA. estrtegia un actuar de excelencia con los pacientes, con otros PS y con el sistema sanitario (Puschel et al., 2017). El dar una atención segura y de calidad a los pacientes está totalmente imbricado con el actuar profesional que todo médico debería tener.

\section{Consideraciones sobre otras competencias relevantes para el desarrollo de la cultura de la seguridad: trabajo en equipo y comunicación}

Para el establecimiento de la cultura de la seguridad se requiere de la interacción de distintas competencias, destacando el trabajo en equipo y la comunicación. Según se señaló previamente, entre 20 a 30\% de los EAs estarían relacionados con problemas de comunicación, liderazgo y/o trabajo en equipo (Leonard et al., 2004). Estas competencias son especialmente importantes en especialidades como la MI. La atención y el cuidado de los pacientes críticos siempre implica a un equipo de PS que debe comunicarse de manera adecuada para optimizar esa atención y cuidado (Dykes et al., 2017).

El Institute of Healthcare Improvement (IHI) ha señalado que el trabajo en equipo y la comunicación son fundamentales en la cultura de la seguridad, permitiendo: (Institute of Healthcare Improvement, 2017):

- Mejorar la toma de decisiones por los equipos, teniendo en cuenta las prioridades en la atención de un paciente.

- Manejar situaciones dinámicas, especialmente situaciones críticas y urgencias médicas.

- Superar o controlar la tendencia natural al error del ser humano.

El IHI ha definido equipo como "un grupo de personas que trabajan juntas de manera coordinada e interdependiente, de manera de maximizar las fortalezas de cada miembro del equipo y lograr un objetivo común". Para lograr estos objetivos, es muy importante que cada miembro del equipo tenga claro su rol y funciones específicas. Además, es fundamental que el trabajo en equipo sea intencional y sistemático. Esta propuesta puede 
enriquecerse con lo señalado por la OMS sobre equipos clínicos en el contexto de la cultura de la seguridad. Según la OMS, un equipo clínico efectivo debe tener las siguientes características: un propósito común, objetivos mensurables, liderazgo eficiente, comunicación efectiva, buena cohesión y respeto mutuo (World Health Organization, 2011).

El IHI y la OMS han reconocido que la comunicación es fundamental para lograr un trabajo en equipo adecuado, señalando que los buenos equipos clínicos comparten ideas e información con rapidez y regularidad, valoran la opinión de cada miembro, llevan registros por escrito y se dan un tiempo de reflexión grupal. La OMS ha planteado usar técnicas específicas y estructuradas que faciliten la comunicación dentro de un equipo. Específicamente para el desarrollo y la mantención de la cultura de la seguridad, la OMS ha propuesto las siguientes medidas para una comunicación efectiva (World Health Organization, 2011):

- Lenguaje crítico: es un conjunto de términos acordados que indican a todos los miembros de un equipo clínico que hay un problema.

- Regla de dos desafíos: un subordinado puede tomar medidas si ha advertido a un superior sobre un problema de seguridad varias veces y este lo ignora.

- Reuniones informativas: sesiones breves y estructuradas en las que el equipo clínico se reúne para hablar sobre un paciente, un procedimiento o una situación.

- Debriefing : este término no tiene una buena traducción al español, consistiendo en una reflexión grupal con énfasis en como optimizar el desempeño de un equipo. Para esto, tras finalizar un evento, el equipo se congrega para un intercambio conciso de opiniones, cuyo objetivo es identificar qué sucedió y qué podría hacerse mejor si se repitiera ese evento.

- SBAR (en español SAER: situación, antecedentes, evaluación, recomendación): es una herramienta de comunicación estructurada que estandariza cómo los miembros del equipo transmiten información crítica.

- Repetición: cuando un mensaje es importante se recomienda repetirlo, esperando una respuesta del receptor que asegure el entendimiento mutuo.

El liderazgo también es relevante para la comunicación efectiva. Se ha planteado que los líderes que ejercen un liderazgo activo son capaces de crear intencionadamente un entorno en el que todo el equipo se siente cómodo al expresar sus inquietudes.

\section{Teorías del aprendizaje a considerar en la formación sobre seguridad asistencial en postgrado}

A continuación, se revisarán algunas teorías del aprendizaje que pueden facilitar la enseñanza-aprendizaje de la SA y de otras competencias importantes para el desarrollo de la cultura de la seguridad. Previo a describir estas teorías y explicar su aplicación en los postgrados de medicina, es importante revisar las características de la educación basada en competencias (Risco de Domínguez, 2014):

- Los aprendices de una determinada disciplina deben cumplir con un perfil de competencias generales y específicas

- La enseñanza-aprendizaje está centrada en el desempeño

- El desarrollo de las competencias es continuo, por niveles y según estándares definidos

- Deben existir suficientes oportunidades de aprendizaje y evaluación, combinando diferentes metodologías y tipos de evaluaciones

- La evaluación de los resultados debe ser sistemática, con el fin de lograr el mejoramiento continuo de los programas y de la formación profesional.

En la enseñanza-aprendizaje de cualquier competencia en postgrado se debe tener en cuenta que se trata de aprendices adultos. Dos modelos de aprendizaje de adultos muy utilizados en la educación de los PS son la Andragogía de Malcolm Knowles (Fasce, 2006) y la Educación Participativa de Jane Vella (Montero, 2007). Ambos hacen énfasis en el aprendizaje centrado en los aprendices, valorando sus experiencias previas y considerando sus necesidades educacionales; estas características los hacen muy aplicables a los postgrados de medicina. En la tabla 2 se resumen los principios en que se basan la Andragogía y la Educación Participativa.

Dos teorías relevantes acerca del aprendizaje de los adultos, en el contexto de la formación de postgrado en medicina, son el aprendizaje experiencial y el aprendizaje social. El aprendizaje experiencial se basa en aprender a través de la experiencia, definiéndose como"aprender a través de la reflexión sobre el hacer" (Yardley et al., 2012). Uno de los principales representantes de esta teoría es David Kolb, quien planteó que el aprendizaje es un proceso en el que una persona crea conocimiento para sí, a través de la transformación de una experiencia. Este autor propuso un modelo cíclico de cuatro etapas de desarrollo del conocimiento: experiencia concreta, observación reflexiva, conceptualización abstracta y experimentación activa. Varios autores han señalado que mediante el modelo de Kolb se puede explicar cómo se produce parte importante del aprendizaje en los postgrados de medicina (Smith et al., 2004). 
Tabla 2: Resumen de los principios de la andragogía y de la educación participativa.

\begin{tabular}{l|l}
\hline El aprendizaje según el modelo de la andragogía & El aprendizaje según el modelo de la educación participativa \\
\hline $\begin{array}{l}\text { Requiere de una concepción personal (el adulto es capaz de establecer } \\
\text { sus necesidades de aprendizaje). }\end{array}$ & Debe estar centrado en la persona. \\
\hline $\begin{array}{l}\text { Debe considerar el rol de la experiencia (la experiencia previa constituye } \\
\text { una base para el nuevo aprendizaje). }\end{array}$ & $\begin{array}{l}\text { Debe considerar las necesidades y temas relevantes para el aprendiz, proyec- } \\
\text { tándolos más allá de la sesión. }\end{array}$ \\
\hline $\begin{array}{l}\text { Debe orientarse a la aplicación (los adultos valoran los aprendizajes } \\
\text { que se vinculan con su quehacer cotidiano). }\end{array}$ & Debe considerar una distribución adecuada del conocimiento. \\
\hline $\begin{array}{l}\text { Debe considerar la motivación intrínseca (los adultos tienen una mayor } \\
\text { tendencia a estar motivados por factores autónomosy metas personales). }\end{array}$ & $\begin{array}{l}\text { Requiere de una planificación y preparación del proceso de enseñan- } \\
\text { za-aprendizaj. }\end{array}$ \\
\hline & $\begin{array}{l}\text { Debe ser participativo (todos los participantes deben tener posibilidades de } \\
\text { escuchary ser escuchados, de ser consultadosy de decidir en algunos aspectos). }\end{array}$ \\
\hline
\end{tabular}

La teoría del aprendizaje social ha sido desarrollada por distintos autores, destacando Lev Semyonovich Vygotsky y Albert Bandura. Esta teoría comprende aspectos del aprendizaje cognitivo y conductual. El aprendizaje conductual presupone que el entorno de las personas causa que estas se comporten de una manera determinada. El aprendizaje cognitivo plantea que los factores psicológicos son importantes influencias en las conductas de las personas. El aprendizaje social sugiere que una combinación de factores del entorno (factores sociales) y psicológicos influyen en la conducta. La teoría del aprendizaje social señala cuatro requisitos para que las personas aprendan y modelen su comportamiento: atención (un evento o conducta debe captar la atención del aprendiz), retención (recordar lo que se ha observado), reproducción (habilidad de repetir la conducta observada) y motivación (querer adoptar esa conducta) (Kay et al.,2016). Otros autores como Etienne Wenger han dado especial relevancia, dentro del aprendizaje social, a los factores del entorno "contexto y comunidad", planteando la importancia de desarrollar "comunidades de aprendizaje" y así potenciar el proceso de enseñaza-aprendizaje (Taylor et al., 2013). Este enfoque podría tener especial sentido para el aprendizaje de la SA en MI. La unidad de cuidados intensivos es un servicio en el que un equipo de salud (comunidad) procura atención y cuidado a los pacientes más graves del sistema sanitario. Este "contexto" de gravedad y de altas exigencias profesionales para esta "comunidad", sin duda, pueden modular el aprendizaje de la SA y de otras competencias.

\section{Metodologías de enseñanza-aprendizaje a considerar en la formación sobre seguridad asistencial en postgrado}

Al revisar en la literatura como se han desarrollado e implementado los dispositivos de aprendizaje sobre SA en postgrado, se encuentra que, en general, consisten en cursos o talleres que combinan actividades teóricas con un importante componente práctico, lo que facilitaría la adquisición de conocimientos y habilidades. Según una revisión sistemática (Kirkman et al., 2015), estos dispositivos varían en duración, desde un par de días hasta varios meses, con una mayor duración de los cursos que consideran equipos multidisciplinarios. Dentro de las metodologías descritas, el aprendizaje en pequeño grupo (APG) ha sido la más frecuentemente usada, destacando la revisión de casos (Case-Based Learning, CBL) y el aprendizaje en equipos (Team-Based Learning, TBL).

El APG es una metodología muy utilizada en pregrado y postgrado en medicina. El APG implica que varios aprendices, habitualmente un máximo de diez, interactúan y trabajan juntos para lograr objetivos comunes de aprendizaje, bajo la conducción de un facilitador. Esta metodología se enmarca dentro del aprendizaje activo y centrado en el aprendiz, a diferencia de otras metodologías como las clases, que en general están centradas en el docente. Otras fortalezas del APG son el grado de autonomía que pueden alcanzar los aprendices y que es una metodología que facilita la reflexión (Edmunds et al., 2010). A continuación, se describirán el TBL y CBL, que son tipos de APG muy utilizados para la formación en SA en postgrado.

La estrategia TBL fue desarrollada a principios de 1990 por Larry Michaelsen, para ser utilizada en un curso de negocios en la Universidad de Oklahoma, difundiéndose posteriormente a otras áreas de la docencia, incluida la medicina. Esta estrategia considera ordenar los contenidos y aprendizajes en ciclos, desarrollándose cada ciclo en dos fases. La fase uno se denomina "Proceso de Aseguramiento del Aprendizaje Inicial" y la fase dos "Actividades de Aplicación" (Parmelee et al., 2010). Es frecuente que, en medicina, por la duración de las sesiones de APG, solo se realice la fase uno, que debería durar entre 45 a 60 minutos. La fase uno del TBL considera los siguientes pasos:

- Lectura previa a la sesión: el facilitador entrega bibliografía para preparar la sesión 
- Prueba individual al inicio de la sesión: los aprendices contestan una breve prueba escrita que evalúa el estudio y la comprensión de la lectura previa

- Prueba en equipo: tras la prueba individual, los aprendices son agrupados en equipos para contestar colaborativamente la misma prueba, lo que permitiría enriquecer sus análisis iniciales y tener retroalimentación inmediata entre los miembros de un mismo equipo

- Corrección y apelación: se corrige la prueba y los distintos equipos pueden apelar en caso de no estar de acuerdo con una o más respuestas. En casos de controversias importantes, los aprendices pueden hacer sus apelaciones por escrito, para posterior revisión por parte del facilitador

- Mini-clase: finalmente se completa el proceso de la fase uno con una mini-clase realizada por el facilitador, para aclarar las dudas de las apelaciones y de los contenidos de la sesión.

La fase dos del TBL implica que todos los equipos trabajen simultáneamente en buscar soluciones a un problema relevante aportado por el facilitador, realizando posteriormente un reporte por equipo y discutiendo las soluciones planteadas en una plenaria.

El CBL o método de casos es un modo de enseñanza en el que los aprendices construyen su aprendizaje a partir del análisis y discusión de experiencias y situaciones de la vida real. El CBL es una estrategia educacional antigua, que fue desarrollada a principios del siglo XX en una escuela de negocios de la Universidad de Harvard. Esta estrategia se usó por primera vez en medicina el 1912 en la Universidad de Edinburgo, por el médico patólogo James Lorrain Smith. Una característica del CBL, que es especialmente interesante en la enseñanza-aprendizaje de la medicina, es que permite que los aprendices relacionen los conocimientos teóricos de un curso con su aplicación práctica, lo que facilitaría el desarrollo del razonamiento clínico. Por esto, algunos autores han planteado que el CBL facilita la preparación de los aprendices para la práctica clínica, mediante el uso de casos clínicos reales. Tras analizar el caso en grupos, los aprendices proponen posibles soluciones fundamentadas en su conocimiento previo. No está definido el tiempo exacto que deben durar las sesiones de $\mathrm{CBL}$, pero en general no duran más de una hora (Thistlethwaite et al, 2012).

CBL y TBL permiten practicar y desarrollar varias competencias transversales, como el trabajo en equipo y la comunicación (Parmelee et al., 2010; Thistlethwaite et al. 2012), que son muy importantes para la Ml y el desarrollo de la cultura de la seguridad. En este sentido, se ha reconocido que un elemento central del aprendizaje con el uso de estas estrategias es que el aprendizaje es colectivo.
Otras metodologías reportadas en la literatura para el aprendizaje de la SA en postgrado son: simulación, mini-clases, estudio personal con apoyo de material audiovisual/material on-line, aprendizaje basado en juegos (gaming), desarrollo de proyectos en grupo en relación a problemas de SA (análisis causa-raíz de una situación y propuesta de un plan de mejora), etc. (Kirkman et al., 2015).

\section{Evaluación del aprendizaje y de los programas educacionales sobre seguridad asistencial}

En el aprendizaje de la SA y en su evaluación, debe considerarse que la seguridad del paciente es una competencia. Para la evaluación del aprendizaje de competencias en medicina, la pirámide de Miller es el modelo más utilizado. Este modelo considera cuatro niveles, partiendo por la evaluación del conocimiento adquirido, que corresponde a la primera etapa en el desarrollo de una competencia, y culminando con la evaluación del desempeño en el contexto clínico. Así, los cuatro niveles de la pirámide de Miller y los instrumentos de evaluación recomendados para cada uno de ellos son (Shumway \& Harden, 2003):

- Sabe (el aprendiz sabe): pruebas escritas y exámenes orales

- Sabe cómo (el aprendiz sabe cómo): pruebas escritas y exámenes orales

- Muestra (el aprendiz muestra): exámenes prácticos y ECOE (Examen de Competencias Objetivo y Estructurado)

- Hace (el aprendiz hace): evaluación en el lugar de trabajo (en $360^{\circ}$, por pares, por docentes, etc.) mediante la observación directa con apoyo de pautas y rúbricas, portafolios, MINI-CEX (Mini Clinical Evaluation Exercise)

Existen varios modelos para evaluar programas educacionales, siendo el modelo de cuatro niveles de Kirkpatrick el más utilizado en la evaluación de programas de formación en SA (Kirkman et al., 2015). El nivel 1 de Kirkpatrick evalúa la satisfacción de los aprendices con el programa educacional. El nivel 2 evalúa la percepción de los aprendices sobre la importancia de lo aprendido durante el programa (nivel 2A), así como la adquisición de conocimientos (nivel 2B). El nivel 3 evalúa los cambios de comportamiento de los aprendices tras realizar el programa educacional. El nivel 4 evalúa como se modifican los resultados de las organizaciones en que se desempeñan los aprendices tras realizar el programa (nivel $4 A)$, junto con variaciones en distintos resultados a nivel de los pacientes, otros PS, estudiantes de pre y postgrado, etc. (nivel 4B). Para evaluar estos distintos niveles, este modelo propone utilizar encuestas, entrevistas, grupos focales, pruebas escritas, ensayos, informes, tareas, sistemas de registros, auto-reportes, estadísticas 
de morbi-mortalidad, etc. (Donald et al., 2006). Es recomendable tener varias evaluaciones por cada uno de los niveles de Kirkpatrick a valorar y utilizar diferentes instrumentos a lo largo del tiempo (antes de la intervención educativa, durante, inmediatamente después y meses/años posterior a su término). Los niveles 1 y 2 son más simples de evaluar, siendo más compleja la valoración de los niveles 3 y 4 . Específicamente el nivel 4B es difícil de evaluar, especialmente en cuanto a los resultados clínicos de los pacientes, pues, junto con la formación de los PS hay múltiples otros factores que inciden en estos resultados.

\section{Formación sobre seguridad asistencial en medicina intensiva}

Los pacientes hospitalizados en una Unidad de Cuidados Intensivos (UCI) son especialmente susceptibles a los incidentes de seguridad. Además de la gravedad de la enfermedad que determinó la hospitalización en $\mathrm{UCl}$, hay que tener presente que los pacientes críticos están bajo el efecto de sedación/analgesia y expuestos a múltiples intervenciones, procedimientos y medicamentos. Estas características particulares permiten explicar los 1,7 errores por paciente por día reportados en UCls de países desarrollados (Kohn et al., 2000), pudiendo ser la situación aún más compleja en países en vías de desarrollo, como Chile. Por otra parte, gran parte de estos errores pueden producir daño significativo o muerte. Ante esto, es clave que los residentes que realicen la subespecialidad de MI tengan una capacitación adecuada en SA, junto con una motivación especial por esta competencia en su quehacer profesional, reconociendo que es igual de importante que otras competencias técnicas.

La preocupación global por la SA y la importancia de su aprendizaje en MI han sido reconocidas por marcos de referencia internacionales sobre formación en postgrado, destacando CanMEDS y COBATRICE. CanMEDS es un marco de referencia desarrollado en la década de 1990 por el Royal College of Physicians and Surgeons of Canada (Frank et al., 2015). Actualmente, es el marco referencial por competencias más difundido y utilizado en el mundo en los postgrados de medicina. El objetivo de CanMEDS es mejorar el cuidado y la atención de los pacientes a través de optimizar el entrenamiento de los residentes. Para ello, este marco referencial ha establecido las competencias necesarias para las distintas especialidades y subespecialidades médicas, con el fin de facilitar la enseñanza-aprendizaje de estas. CanMEDS propone siete roles que todo residente debe desarrollar en el transcurso de su programa de especialidad o subespecialidad.
Estos roles son: erudito, comunicador, colaborador (capacidad de trabajo en equipo), defensor/promotor de la salud, profesional, líder y experto médico; requiriendo este último rol el desarrollo armónico de los otros. Para cada rol se establecen competencias y para cada competencia hitos o milestones. Estos últimos corresponden al nivel esperado de progreso de una competencia en un determinado momento de la formación de postgrado, ya que las competencias se van adquiriendo en forma progresiva desde novato a maestro. Respecto a la formación de los futuros intensivistas, CanMEDS ha establecido las competencias que estos deben alcanzar sobre SA dentro de los roles de líder, promotor y experto médico, lo que se detalla en la tabla 3 (Royal College of Physicians and Surgeons of Canada, 2014). Sin embargo, impresiona que, para el establecimiento de la cultura de la seguridad en MI se necesita del desarrollo de los siete roles de CanMEDS. Los futuros especialistas en MI deben conocer los mejores estándares de atención (erudito), velar por una buena comunicación y trabajo en equipo para evitar EAs, promover las prácticas seguras (promotor), demostrar profesionalismo y liderazgo en reconocer errores y desarrollar planes de mejora, transformándose así en expertos médicos en SA. Debido a esto, el marco referencial de CanMEDS ofrece un andamiaje que puede ayudar a facilitar la implementación de programas educacionales sobre SA en MI.

COBATRICE (Competency-Based Training in Intensive Care Medicine in Europe) es un marco referencial por competencias exclusivo para MI. Este marco fue desarrollado por la Sociedad Europea de Medicina Intensiva en el 2006, siendo su propósito homogeneizar el aprendizaje de la MI, haciendo énfasis en el aprendizaje reflexivo. Para esto, COBATRICE ha establecido un conjunto de conocimientos, habilidades y actitudes que los residentes deben desarrollar durante su formación, organizándolos en 12 dominios que consideran 102 competencias. Estos 12 dominios son: resucitación y manejo inicial del paciente grave, diagnóstico y evaluación, manejo de la enfermedad aguda y de las fallas orgánicas, intervenciones terapéuticas y soporte orgánico, procedimientos, atención perioperatoria, confort y recuperación, atención a pacientes terminales, atención pediátrica, transporte, seguridad del paciente y manejo de los sistemas de salud, y por último, profesionalismo. En la tabla 3 se muestran las competencias sobre SA que un residente debe alcanzar durante su formación según este marco referencial, las que corresponden al dominio seguridad del paciente y manejo de los sistemas de salud (Sociedad Europea de Medicina Intensiva, 2006). 
Tabla 3: Resumen de las competencias asociadas a seguridad asistencial en la formación de los intensivistas según CanMEDS y COBATRICE.

\begin{tabular}{|c|c|c|}
\hline Marco Referencial & Rol/Dominio & Competencias esperables al finalizar el programa \\
\hline & Líder & Participar en los procesos de mejora y evaluación sistémica de la de la SA. \\
\hline \multirow[t]{3}{*}{ CanMEDS } & Líder & Identificar, analizar y minimizar el riesgo de EA. \\
\hline & $\begin{array}{l}\text { Promotor y Defensor de } \\
\text { la Salud }\end{array}$ & Describir el rol de la profesión médica en abogar por la salud y la SA. \\
\hline & Experto Médico & $\begin{array}{l}\text { Implementar prácticas de mejora continua de la calidad, como guías y prácticas basadas en la } \\
\text { evidencia. }\end{array}$ \\
\hline \multirow[t]{4}{*}{ COBATRICE } & \multirow{4}{*}{$\begin{array}{l}\text { Seguridad del paciente } \\
\text { y manejo de los sistemas } \\
\text { de salud }\end{array}$} & Identificar los peligros ambientales y promover la seguridad para los pacientes y el personal. \\
\hline & & $\begin{array}{l}\text { Identificar y minimizar el riesgo de incidentes críticos y EA, incluidas las complicaciones de una } \\
\text { enfermedad crítica. }\end{array}$ \\
\hline & & Organizar la presentación sobre casos de EA. \\
\hline & & Evaluar críticamente y aplicar pautas, protocolos y paquetes de atención (bundles). \\
\hline
\end{tabular}

Abreviaciones: SA (seguridada asistencial), EA (evento adverso).

\section{Experiencia piloto sobre formación en seguridad asistencial para residentes de medicina intensiva del adulto de la Pontificia Universidad Católica de Chile}

El programa de postgrado en MI de la UC es actualmente el más antiguo de Chile. De acuerdo a su sílabo, este programa tiene como propósito "educar a los médicos de modo que al final de su período de formación posean los niveles de competencia necesarios para el ejercicio independiente de la especialidad y sean capaces de desarrollar continuamente su autoformación". Además, el programa define como competencias esperadas al término de los dos años de formación, que "el residente demuestre un manejo integral de la UCl a nivel de experto, dirigiendo la visita, la toma de decisiones, la resolución de casos complejos, el enfrentamiento con la familia, la relación con pares de otras especialidades y el equipo de salud, desarrollando cualidades de liderazgo" (Programa de Especialidad Derivada en Medicina Intensiva Del Adulto, 2010).

Dada la importancia de la SA en MI, es fundamental asegurar un aprendizaje curricular de esta, y así evitar un aprendizaje oportunista de esta competencia tan importante para el rol profesional de los intensivitas. Por esto que, en el 2018, con aprobación del jefe de programa y comité de programa de $\mathrm{MI}$, se realizó una actividad piloto de formación en SA para los residentes de MI del adulto de la UC. Esta actividad consistió en un taller presencial de cinco sesiones y su propósito fue proveer los conocimientos y las habilidades necesarias para que los residentes de MI lograran una experticia adecuada en SA. La tabla 4 muestra los objetivos, contenidos y metodologías en cada una de las sesiones. Este taller contó con un equipo multidisciplinario de facilitadores especialistas en MI (dos médicas intensivistas, una enfermera y un químico farmacéutico especialista en farmacia clínica). Además, al momento de realizar el taller las dos médicas intensivistas estaban cursando el Magíster en Educación Médica (MEM) de la UC.

\section{Métodos}

Para el taller se utilizaron metodologías de aprendizaje en pequeño grupo (Edmund et al., 2010), como TBL, CBL, aprendizaje basado en juegos o gaming, etc. Para esto, en la primera sesión del taller los facilitadores dividieron a los 18 residentes del programa en tres grupos, manteniéndose estos grupos de trabajo durante todo el taller. En las tres últimas sesiones los residentes trabajaron con casos de EAs, realizando los respectivos reportes, análisis causa-efecto y desarrollando un plan de mejora para cada uno de estos; lo que fue presentado en la última sesión.

Para la evaluación del aprendizaje se consideraron evaluaciones formativas y sumativas, evaluándose todas las actividades del taller. De acuerdo con la Pirámide de Miller (Shumway \& Harden, 2003), para la evaluación de los objetivos de aprendizaje de este taller, que consideraban la adquisición de conocimientos junto con su aplicación y análisis, se usaron pruebas de selección múltiple y términos pareados, al igual que la aplicación de pautas. Las pruebas se utilizaron para evaluar el TBL y para las otras actividades se usaron pautas de cotejo y pautas de apreciación global. Estos instrumentos de evaluación del aprendizaje fueron desarrollados con el apoyo de docentes MEM UC.

Al realizarse las distintas actividades del taller en grupos pequeños, los residentes también pudieron practicar las otras competencias necesarias para el desarrollo de la cultura de la seguridad, como la comunicación, el trabajo en equipo, el profesionalismo y el liderazgo. De estas competencias transversales, se dio especial 
énfasis a la comunicación, en las actividades de reporte de EAs y en las de métodos de mejora continua (análisis causa-raíz, análisis causa-efecto y elaboración de planes de mejora). Para esto, se utilizó la herramienta de comunicación estructurada SBAR o SAER (situación, antecedentes, evaluación y recomendación), que ha sido específicamente recomendada para comunicación de incidentes de seguridad y EAs.

Este programa piloto de formación en SA, para residentes de MI, se evaluó mediante el modelo de evaluación de programas educacionales de Kirkpatrick (Donald et al., 2006). Específicamente, se revisaron los niveles 1 (satisfacción), 2A (percepción) y 2B (adquisición de conocimientos). Los niveles 3 y 4 de Kirkpatrick están pendientes de evaluar, pues, según se señaló previamente, su valoración es más compleja. La satisfacción y percepción se evaluaron mediante encuestas de apreciación global contestadas por los residentes, considerando la encuesta de satisfacción diez enunciados y la de percepción siete. En ambas, los enunciados podían ser calificados de uno a cinco, correspondiendo uno a "muy en desacuerdo" y cinco a "muy de acuerdo". La encuesta de satisfacción se aplicó después de la primera y última sesión del taller, y la de percepción antes del inicio y tras el término del taller. Los resultados entre las encuestas iniciales y finales se compararon mediante el Test de Fischer, considerando un valor $\mathrm{P}<0,05$ como estadísticamente significativo. Para la evaluación del nivel $2 \mathrm{~B}$, se consideraron las pruebas del TBL de la primera sesión del taller (prueba individual y grupal), y las pautas de apreciación global que se aplicaron a las actividades de trabajo grupal realizadas en las 3 últimas sesiones.

Tabla 4: Objetivos, contenidos y metodologías del taller de seguridad asistencial para residentes de medicina intensiva.

\begin{tabular}{|c|c|c|c|}
\hline Objetivos & Contenidos & Metodologías & Sesión \\
\hline $\begin{array}{l}\text { Identificar los componentes de la SA. } \\
\text { Describir el factor humano y su } \\
\text { importancia en SA. }\end{array}$ & $\begin{array}{l}\text { Error, riesgo, seguridad del paciente, incidente de seguridad, } \\
\text { EA, evento centinela, víctimas de la SA, cultura de la seguridad. } \\
\text { Teoría del error, factor humano. }\end{array}$ & $\begin{array}{l}\text { Estudio personal previo } \\
\text { TBL }\end{array}$ & Primera \\
\hline $\begin{array}{l}\text { Distinguir en la práctica diaria la } \\
\text { ocurrencia de incidentes de seguridad. } \\
\text { Identificar los elementos más im- } \\
\text { portantes de la gestión del riesgo. }\end{array}$ & $\begin{array}{l}\text { Error en la práctica clínica, incidente de seguridad, EA, evento } \\
\text { centinela, víctimas de la SA. } \\
\text { Identificación y prevención del riesgo (primaria, secundaria, } \\
\text { terciaria), niveles de gestión (clínica, mesogestión, macrogestión). }\end{array}$ & $\begin{array}{l}\text { Estudio personal previo } \\
\text { Análisis de videos } \\
\text { Gaming } \\
\text { Mini-clase }\end{array}$ & Segunda \\
\hline $\begin{array}{l}\text { Distinguir las prácticas clínicas se- } \\
\text { guras. } \\
\text { Elaborar reportes de incidentes y EA. }\end{array}$ & $\begin{array}{l}\text { Prácticas clínicas seguras, errores de medicación, SA y proce- } \\
\text { dimientos invasivos, manejo del riesgo clínico. } \\
\text { Sistemas de reportes de EA. }\end{array}$ & $\begin{array}{l}\text { Estudio personal previo } \\
\text { CBL } \\
\text { Mini-clase } \\
\text { Ejercicios reporte EAs (trabajo } \\
\text { grupal)* }\end{array}$ & Tercera \\
\hline $\begin{array}{l}\text { Realizar análisis causa-raíz en casos } \\
\text { de EA. } \\
\text { Utilizar la herramienta de comunica- } \\
\text { ción estructurada SAER en el reporte } \\
\text { y análisis de EA. }\end{array}$ & $\begin{array}{l}\text { Análisis causa-raíz (ACR), diagrama de Ishikawa (causa-efecto), } \\
\text { ciclo de Deming (planificar-hacer-verificar-actuar), métodos } \\
\text { de mejora continua. } \\
\text { SAER (situación, antecedentes, evaluación y recomendación). }\end{array}$ & $\begin{array}{l}\text { Estudio personal previo } \\
\text { Análisis de videos } \\
\text { Ejercicios de diagrama cau- } \\
\text { sa-efecto (trabajo grupal) * } \\
\text { Mini-clase }\end{array}$ & Cuarta \\
\hline $\begin{array}{l}\text { Diseñar un plan de mejora en base a } \\
\text { los análisis de incidentes de seguridad. }\end{array}$ & $\begin{array}{l}\text { Análisis causa-raíz (ACR), métodos de mejora continua (plan } \\
\text { de mejora). }\end{array}$ & $\begin{array}{l}\text { Exposición grupal (análisis } \\
\text { caso de EA y plan de mejora)* }\end{array}$ & Quinta \\
\hline
\end{tabular}

Abreviaciones: SA (seguridada asistencial), EA (evento adverso), TBL (team-based learning), CBL (case-based learning)

*Las actividades en grupo culminan con una presentación en la última sesión

\section{Resultados y discusión}

Los 18 residentes del programa participaron y aprobaron el taller, con una calificación promedio de 6,5 20,2, siendo el 7 la calificación máxima. Todos los residentes contestaron las cuatro encuestas.
El $88 \%$ y $67 \%$ de los enunciados de las encuestas iniciales de satisfacción y percepción, respectivamente, fueron evaluados positivamente (de acuerdo o muy de acuerdo), mejorando estos resultados en las encuestas finales. En la encuesta final de satisfac 
ción, se obtuvo $100 \%$ de respuestas positivas; y en la de percepción $76 \%$ de los enunciados fueron calificados de acuerdo o muy de acuerdo. Sin embargo, la mejoría en los resultados no alcanzó la significación estadística.

Estos resultados son similares a otras experiencias sobre formación en SA, que también consideraron la implementación de talleres (Putnam et al., 2016). Aunque la adquisición de conocimientos por parte de los residentes de Ml fue adecuada, de acuerdo con las calificaciones obtenidas, preocupa que no haya habido una mejoría significativa en la percepción de la importancia de la SA en UCl. Sin embargo, según se mencionó anteriormente, se ha descrito la falta de reconocimiento por parte de los médicos que la SA es esencial en su rol profesional (Vivekananda-Schimdt et al.,2016). Esto es algo que debe revisarse, tanto en la formación general de los médicos, y especialmente en áreas como la MI. Es esperable que, si un médico no le da importancia a una competencia, no la aprenda ni la considere necesaria en su trabajo cotidiano.

En literatura se encontró que las iniciativas educacionales dirigidas al aprendizaje de la SA que han logrado resultados positivos, incluyendo la mejoría en la percepción sobre la importancia de esta competencia, han sido las que han combinado actividades teórico-prácticas, a modo de talleres, con aprendizaje en el lugar de trabajo (Slater et al.,2012). El aprendizaje en el lugar de trabajo se ha definido como el proceso de adquirir conocimientos y habilidades mediante el contacto real con pacientes en el ambiente clínico, enfocándose en la solución de problemas de salud. Estos resultados favorables de los dispositivos que incluyeron aprendizaje en lugar de trabajo, pueden explicarse de distintas formas, lo que excede a esta revisión narrativa. Cabe destacar que varios autores han señalado que el ambiente clínico sería el mejor lugar para el aprendizaje de varias competencias fundamentales para la cultura de la seguridad, como la comunicación, el profesionalismo y el trabajo en equipo (Ramani \& Leinster, 2008). Por otra parte, dentro de las teorías que han tratado de explicar cómo aprenden los adultos, el "aprender a través de la reflexión sobre el hacer" del aprendizaje experiencial podría lograrse más fácilmente en el entorno clínico.

Ante los resultados obtenidos tras evaluar esta experiencia piloto sobre formación en SA, para residentes de MI de la UC, en conjunto con el jefe de este programa de subespecialidad y con el comité de programa, se decidió agregar a este taller un módulo de aprendizaje en el lugar de trabajo. Así, el taller se realizará durante el primer año de la subespecialidad, y en el segundo año, durante las rotaciones clínicas, habrá un módulo de aprendizaje de SA en el ambiente clínico de la UCl. En este segundo módulo, junto con el aprendizaje en el lugar de trabajo, se considerarán otras metodologías que pueden facilitar el aprendizaje de distintas competencias en el entorno clínico, como el modelaje, la práctica deliberada y la práctica reflexiva (Côté \& Laughrea 2014; Ericsson, 2004). Además, el realizar actividades enfocadas al aprendizaje de la SA en el contexto clínico, permitirá la interacción de los residentes de $\mathrm{MI}$ con los otros miembros del equipo de salud, favoreciendo el aprendizaje interprofesional, lo que constituía una de las falencias del taller. La versión mejorada de este dispositivo, que se espera implementar entre 2020 y 2022, también será evaluada tras su realización mediante el modelo de Kirkpatrick.

Por último, de acuerdo a la revisión de la literatura nacional, que incluyó la búsqueda en libros de resúmenes de congresos, esta ha sido la primera experiencia piloto sobre enseñanza-aprendizaje de SA para residentes de MI en nuestro país.

\section{Conclusión}

Actualmente se considera que la SA es un componente esencial de una atención de calidad, siendo un requisito básico para cualquier actividad clínica, por lo que, es necesaria la formación de los médicos en esta. Además, la capacitación en SA tiene un impacto positivo a nivel de la reducción de errores y eventos adversos.

La necesidad de la enseñanza-aprendizaje de la SA en medicina intensiva ha sido establecida por marcos de referencia internacionales sobre formación de postgrado, por lo cual, esta competencia debe ser considerada en los distintos programas de esta subespecialidad en Chile. En este contexto, se desarrolló un taller piloto dentro del Programa de Residencia de Medicina Intensiva del Adulto de la Pontificia Universidad Católica de Chile. Al evaluar este dispositivo educacional, no se encontró una mejoría significativa en la percepción sobre la importancia de la SA por parte de los residentes. Ante esto, se desarrollará una versión mejorada de este dispositivo de formación en SA, que considerará actividades teórico-prácticas junto con aprendizaje en el lugar de trabajo en el ambiente clínico de la UCI.

\section{Contribuciones y reconocimiento}

Sin fuentes de financiamiento. Ni Carolina Ruiz ni Denisse Zúñiga tienen conflictos de interés a declarar. Este artículo se basa en una tesis de magíster en Educación Médica. Carolina Ruiz fue la tesista y Denisse Zúñiga la profesora guía.

\section{Referencias}

Bagian J. (2005). The Future of Graduate Medical Education: A Systems-Based Approach to Ensure Patient Safety. Acad Med 90,1199-1202. 
Côté L \& Laughrea PA (2014). Preceptors' Understanding and Use of Role Modeling to Develop the CanMEDS Competencies in Residents. Academic Medicine 2014 89,934-939.

Couceiro A \& Muñoz M. (2008). El perfil de la enseñanza de la bioética: ¿médico virtuoso o médico capacitado?. Rev Educ Cienc Salud 5, 82-91.

Donald L. Kirkpatrick D \& Kirkpatrick J. (2006). Evaluating Training Programs, Third edition. Berrett-Koehler Publishers, San Francisco.

Dykes P, Rozenblum R, Dalal A, Massaro A \& Chang F. (2017). Prospective Evaluation of a Multifaceted Intervention to Improve Outcomes in Intensive Care: The Promoting Respect and Ongoing Safety Through Patient Engagement Communication and Technology Study. Crit Care Med 45,e806-e813.

Edmunds S \& Brown G. (2010). Effective small group learning: AMEE Guide No. 48, Medical Teacher 32, 715-726.

Ericsson KA. (2004). Deliberate practice and the acquisition and maintenance of expert performance in medicine and related domains. Acad Med 79,570-81.

Fasce E. (2006). Andragogía. Rev Educ Cienc Salud 3,69-70.

Flott K, Fontana G, Dhingra-Kumar N, Yu A, Durkin M \& Darzi A. (2017). Health care must mean safe care: enshrining patient safety in global health. Lancet. 89,1279-1281.

Frank JR, Snell L \& Sherbino J. 2015. CanMEDS 2015 Physician Competency Framework. Ottawa: Royal College of Physicians and Surgeons of Canada; 2015. Accedido en: http://:www.royalcollege. $\mathrm{ca} / \mathrm{rcsite} /$ documents/canmeds/canmeds-full-framework/ el 5 mayo de 2020.

Institute of Healthcare Improvement (2017). Open School Course Summary Sheet/PS 104:Teamwork and Communication in a Culture of Safety. Accedido en: https://www.quizlet.com/348960725/ps104-teamwork-and-communication-in-a-culture-of-safety-flashcards/ el 5 mayo de 2020.

Kay D \& Kibble J. (2016). Learning theories 101: application to everyday teaching and scholarship. Adv Physiol Educ 40,17-25.

Kirkman MA, Sevdalis N, Arora S, Baker P, Vincent Ch \& Ahmed M et al. The outcomes of recent patient safety education interventions for trainee physicians and medical students: a systematic review. BMJ Open 5,e007705.
Kohn LT, Corrigan JM, Donaldson MS \& Committee on Quality of Health Care in America, Institute of Medicine. (2000). To Err is Human: Building a Safer Health System. Accedido en: http://www. nap.edu/catalog/9728.html/ el 5 mayo de 2020.

Lancis-Sepúlved M \&Asenjo-Araya C. (2014). Estudio de incidencia de eventos adversos en una clínica privada en Chile. Rev Calid Asist 29,78-83.

Leonard M, Graham S \& Bonacum D. (2004). The human factor: the critical importance of effective teamwork and communication in providing safe care. Qual Saf Health Care 13,i85-i90.

Ley 20.584. (2012). Regula los derechos y deberes que tienen las personas en relación a las acciones vinculadas a su atención en salud. Diario Oficial de Chile 40245,1-6.

Manser T. (2009). Teamwork and patient safety in dynamic domains of healthcare: a review of the literature. Acta Anaesthesiol Scand 53,143-51.

Montero J. (2007). Educación participatica de adultos. El modelo dialogante de Jane Vella. Experiencia en Chile. Ars Medica 3,1-12.

Organización Mundial de la Salud. (2019). Medidas Mundiales en materia de seguridad del paciente. Consejo Ejecutivo de la Organización Mundial de la Salud, 144 reunión enero 2019. EB144/CONF./8. Accedido en: http://www.apps.who.int/gb/ebwha/pdf_files/EB144/ B144_CONF8-sp.pdf/ el 5 mayo de 2020.

Parmelee D \& Michaelsen L. (2010). Twelve tips for doing effective Team-Based Learning (TBL). Medical Teacher 32, 118-122.

Pontificia Universidad Católica de Chile, Dirección de Postgrado de la Escuela de Medicina. (2010). Programa de Especialidad Derivada en Medicina Intensiva Del Adulto. Accedido en: https://www.medicina.uc.cl/postgrado/especialidades-medicas/ especialidades derivadas/medicina-intensiva-del-adultos/ el 5 mayo de 2020

Puschel K, Repetto P, Bernales M, Barros J, Perez I \& Snell L. (2017). "In our own words": Defining medical professionalism from a Latin American perspective. Educ Health 30,11-8.

Putnam L, Ostovar-Kermani T, Alawadi Z, Etchegaray J, Ottosen M \& Thomas E. (2016). How Should Surgical Residents Be Educated About Patient Safety: A Pilot Randomized Controlled Trial. J Surg Educ 73,660-667.

Ramani S \& Leinster S. (2008). AMEE Guide no. 34: Teaching in the clinical environment. Medical Teacher 30,347-64. 
Risco de Domínguez G. (2014). Diseño e implementación de un currículo por competencias para la formación de médicos. Rev Peru Med Exp Salud Pública 31, 572-81.

Royal College of Physicians and Surgeons of Canada. (2014). Objectives of Training in the Subspecialty of Adult Critical Care Medicine. Accedido en: http://www.royalcollege.ca/rcsite/documents/ibd/ critical_care_adult_otr_e.pdf/ el 5 mayo de 2020

Ruiz C. (2019). Diseño de un dispositivo de aprendizaje en seguridad asistencial de pacientes críticos para el Programa de Residencia de Medicina Intensiva del Adulto de la Escuela de Medicina de la Pontificia Universidad Católica de Chile. Tesis del Magíster Educación Médica. Accedido en: https://www.repositorio.uc.cl/ handle/11534/26920/ el 5 mayo de 2020

Shumway JM \& Harden RM. (2003) AMEE Guide No. 25: The assessment of learning outcomes for the competent and reflective physician. Medical Teacher 25,569-584.

Slater B, Lawton R, Armitage G, Bibby J \& Wright J. (2012). Training and Action for Patient Safety: Embedding Interprofessional Education for Patient Safety Within an Improvement Methodology. $J$ Contin Educ Health Prof 32,80-89.

Smith CS, Morris M, Francovich C, Hill W \& Gieselman J. (2004). A qualitative study of resident learning in ambulatory clinic. The importance of exposure to 'break-down' in settings that support effective response. Adv Health Sci Educ Theory Pract 9,93-105.

Sociedad Europea de Medicina Intensiva (2006). Capacitación basada en competencias en Medicina Intensiva. Programa COBATRICE. Accedido en: http://www.cobatrice.org/Data/upload/images/File/ About/Cobaresources/CoBaTrICE_Syllabus.pdf/ el 5 mayo de 2020.
Superintendencia de Salud de Chile. (2017). Resultados de Fiscalización de los Derechos en Salud. Accedido en: http://www. supersalud.gob.cl/observatorio/671/articles-16230_recurso_8. pdf./ el 5 mayo de 2020

Taylor D \& Hamdy H. (2013). Adult learning theories: Implications for learning and teaching in medical education: AMEE Guide No. 83. Medical Teacher 35, e1561-e1572.

Thistlethwaite J , Davies D, Ekeocha S, Kidd J, MacDougall C \& Matthews P et al. (2012) The effectiveness of case-based learning in health professional education. A BEME systematic review: BEME Guide No. 23. Medical Teacher 34, e421-e444.

Vivekananda-Schmidt P \& Sandars J. (2016). Developing and implementing a patient safety curriculum. Clin Teach.13,91-7.

World Health Organization. (2009). International Classification for Patient Safety Statement of Purpose. Accedido en: https://www. who.int/patientsafety/taxonomy/icps_full_report.pdf/el 5 mayo de 2020.

World Health Organization. (2011). The multiprofessional patient safety curriculum guide, Topic 4: Being an effective team player. Accedido en: https://www.who.int/patientsafety/education/curriculum/who_mc_topic-4.pdf/ el 5 mayo de 2020

World Health Organization. (2017). Patient safety: making health care safer. Accedido en: https://www.who.int/iris/handle/10665/255507/ el 5 mayo de 2020

Yardley S, Teunissen P \& Dornan T. (2012). Experiential learning: AMEE Guide No. 63. Medical Teacher 34,102-e115. 